\title{
Ospemifene plus fractional CO2 laser: a powerful strategy to treat postmenopausal vulvar pain
}

\author{
F. Murina, R. Felice, S. Di Francesco, L. Nelvastellio \& I. Cetin
}

Lower Genital Tract Disease Unit, V. Buzzi Hospital, University of Milan, Milan, Italy

CONTACT Filippo Murina filippomurina577@gmail.com Lower Genital Tract Disease Unit, V. Buzzi Hospital, University of Milan, Via Castelvetro 32, Milan, Italy

\begin{abstract}
This study is a single-center, retrospective analysis of postmenopausal women presenting with dyspareunia and vulvar pain, aiming to evaluate relative effectiveness of vestibular CO2 laser therapy as a treatment. Three monthly sessions of laser were performed to each patient and thereafter a three-months follow-up was stablished. A total number of 72 patients undergoing vestibular laser treatment were recruited from patient files in the period between 2016 and 2018. Among these, 39 women also received a concomitant treatment with ospemifene $(60 \mathrm{mg} /$ day) during the study period. There was a statistically significant reduction of all the symptoms in both groups up to the three month follow-up. Regarding dryness and dyspareunia, the relief tent to be more prominent in the ospemifene+laser group at all followups and remained statistically significant at three-month follow-up. Specifically, vestibular dryness was significantly lower in the ospemifene+laser group compared with the laser treatment group ( $-87 \% \mathrm{vs}-34 \%$, respectively), and the vestibular health score started declining faster in the ospemifene+laser group. Although, additional research is needed to understand the mechanism of action, our data shows that a combination regimen of laser and ospemifene may improve clinical effectiveness for long-term treatment of symptoms associated with the under-recognized genitourinary syndrome of menopause.
\end{abstract}

\section{KEYWORDS}

genitourinary syndrome of menopause; vulvar and vaginal atrophy; vestibular CO2 laser therapy; ospemifene; dyspareunia; vulvar pain 


\section{Introduction}

Vulvar and vaginal atrophy (VVA), a chronic postmenopausal health condition that occurs because of a hypoestrogenic state, is common yet often underreported [1]. In untreated postmenopausal women, the incidence of VVA symptoms, such as vaginal dryness, itching, burning, and dyspareunia, is estimated to be approximately $60 \%$ [2]. In 2014, genitourinary syndrome of menopause (GSM) has been accepted as a consensus new terminology for vulvovaginal atrophy and defined as a collection of symptoms and signs associated with a decrease in estrogen and other sex steroids syndrome, including but not limited to genital symptoms of dryness, burning, and irritation; sexual symptoms of lack of lubrication, discomfort or pain, and impaired function; and urinary symptoms of urgency, dysuria, and recurrent urinary tract infections [3]. GSM is characterized by changes in the quantity and quality of vaginal secretions, loss of collagen, the vaginal walls become thinner, less elastic, and pale with loss of rugation; the vaginal surface becomes friable with petechiae, ulcerations, and bleeding often occurring after minimal trauma [4].

Current treatment options for GSM consist of systemic hormone therapy, vaginal estrogen products, dehydroepiandrosterone (DHEA) and over-the-counter nonhormonal lubricants and moisturizers [5].

Local estrogen preparations in the form of tablets, rings, or creams are often prescribed as they are perceived to have a low systemic absorption and have been shown to result in significant symptomatic benefit [6,7]. However, there are some limitations to these therapies, such as the fact that incorrect dose administration, leakage, and mess of vaginal formulations may affect the use of creams, moisturizers and lubricants. For these reasons, some women may prefer oral treatments over vaginal therapies due to ease of use. In clinical practice, it is common that the cause of symptoms like painful penetration and dryness is mainly located in the vestibular area, and they fail to improve when being treated with intravaginal preparations such as the vaginal tablet. It is possible that local effects of estrogen may vary, based on the location of absorption. In fact, doppler flow studies showed preferential delivery toward the uterus when a vaginal tablet was placed in the inner third of the vagina, and preferential delivery toward periurethral areas when placed in the outer one third of the vagina [8].

Ospemifene is an oral estrogen receptor agonist/antagonist (SERM) that has tissue-specific estrogenic or antiestrogenic effects, acting selectively as an estrogen receptor agonist on the vulva and vagina [9]. It was also confirmed the safety of ospemifene for up to one year, finding no significant estrogenic or clinically relevant adverse effects on the endometrium or the breast [10]. The use of vaginal laser therapy is one of the new non-hormonal therapeutic approaches for the management of postmenopausal women with clinical signs and symptoms of GSM [11]. 
The fractional laser used for vaginal epithelial resurfacing activates heat shock proteins that in turn activate growth factors, resulting in an increase in vascularity, collagen, extracellular matrix production, and thickness of vaginal epithelium [12].

This is the reason why in recent times new treatments that work on the long-term and on the level of connective tissue and vascularization are being developed, some of which represent important alternatives. This study focused on the evaluation fractional $\mathrm{CO} 2$ vestibular laser treatment combination with oral ospemifene in postmenopausal women presenting with dyspareunia and vulvar pain.

\section{Methods}

The study was a single-center, retrospective analysis of women who underwent vestibular CO2 laser therapy as a treatment of postmenopausal dyspareunia and vulvar pain. The used data were obtained from patient files of women diagnosed with this condition in the period between 2016 and 2018. Among these were identified women who received a 4-week pretreatment with ospemifene (60 mg/day), which has been extended during the three sessions of laser performed to each patient and thereafter for the three months follow-up. Review Board approval for the study was obtained and all participating individuals gave written informed consent. Signed written consent was also obtained for use clinical photographs.

Inclusion criteria were postmenopausal women presenting with vulvar pain and/or burning plus dyspareunia related to GSM and vestibular atrophy (thinned, dry, fragile, or pale mucosa), in whom previous therapeutic intervention (medical, hormonal, and behavioral) was unsuccessful or produced unsatisfactory outcomes. Exclusion criteria included presence of active genital infection, moderate to severe hypertonic pelvic floor dysfunction or overactivity (as determined by physician digital examination), use of hormone therapy (either systemic or topical) within the last 60 days prior to the initial assessment, a current or past thromboembolic or blood coagulation disorder or clinically significant findings at physical examination. Women allergic to the test drug or its constituents and subjects with a serious disease or chronic condition were also excluded. The fractional microablative CO2 laser (Smart Xide2; V2LR Monnalisa Touch System, DEKA Florence, Italy) was administrated within a protocol providing three time-points of application at baseline (T1), at 30 days (T2) and at 60 days (T3), equipped with an appropriate probe for the vulva; the probe has two parallel arms positioned at a distance of approximately $2 \mathrm{~cm}$. Settings were 30-W power, a dwell time of 1,000 ls, DOT spacing of 700 lm, and SmartStack 2 using DP pulse mode, parameters adjusted according to our previous study [13]. 
A lidocaine $2.5 \%$ and prilocaine $2.5 \%$ cream was applied at the vulvar vestibule five minutes prior to the procedure. During and after the laser intervention, patient discomfort, and treatment tolerability, as well as potential adverse events related to ospemifene were monitored. Patients were recommended to avoid coital sexual activity for at least seven days after laser treatment.

At the first assessment, symptoms of dryness, burning/pain and dyspareunia were evaluated on a 10 $\mathrm{cm}$ visual analog scale (VAS). The scale's left extremity indicates the complete absence of symptoms (0) and the right extremity indicates the worst possible symptom.

Visual examination of the vulvar vestibule was also conducted (vestibular health score), which included observations for petechiae, pallor, friability, dryness, and redness in the mucosa. Ratings were based on a four-point scale ( 0 , none; 1 , mild; 2 , moderate; 3 , severe). Data were collected at baseline and at weeks 4, 8, and 12 after treatment and at final follow-up at three months.

All the results were reported as the mean standard error of absolute values. Baseline values were compared by Student's t-test. To determine the changes in dyspareunia and the appearance of vulvar vestibule scores during the treatment and at the end of follow-up compared to the baseline, the Mann-Whitney U-test was performed. Treatment differences were expressed as least squares means (standard error) and 95\% confidence intervals (CIs), and the statistical significance was defined as $\mathrm{p}<.05$. Statistical analysis was performed with SPSS version 17.0 for Windows (IBM SPSS, Armonk, NY).

\section{Results}

A total of 72 patients undergoing vestibular laser treatment for GSM symptoms were recruited in this study, among these were identified 39 women who received during the study period a treatment with ospemifene (60 mg/day). Patient's demographic data are included in Table 1.

There were no statistically significant differences between the groups' (ospemifene+laser group and laser group) demographic data. There was a statistically significant $(\mathrm{p}<.01)$ reduction of all the symptoms in both groups up to the three month follow-up; however, the relief symptoms was more prominent in the ospemifene+laser group at all follow-ups (after three laser sessions and 3 month follow-up) regarding dryness and dyspareunia (Table 2).

In particular, the improvement of dryness and dyspareunia in the ospemifene+laser treatment remained statistically significant higher compared with the laser group at three-month follow-up (Figure 1). The largest difference between the two groups was related to vestibular dryness, in fact was shown to be significantly lower in the ospemifene+laser group compared with the same symptom in the other treatment group ( $-87 \%$ vs. $-34 \%$ respectively). 
The vulvar pain relief instead showed a similar reduction in the two study groups (Figure 1). The vestibular health score improved significantly in both groups, but in the ospemifene+laser group it started declining faster (Table 3 and Figure 2).

Side effects included a sensation of mild-to-moderate pain in 12 patients (16.6\%), as well as slight transient edema after laser treatment (6\% in ospemifene+laser group and $8 \%$ in laser group). In the ospemifene+laser group, four of patients (10.2\%) experienced hot flashes but this did not led to a discontinuation of therapy.

\section{Discussion}

This study provides evidence of the effectiveness and tolerability of oral ospemifene and vestibular fractional CO2 laser for treating symptoms of GSM, particularly in women who report dyspareunia vulvar pain and dryness as their bothersome symptoms. The main contribution of this study is the first-time comparison of fractional $\mathrm{CO} 2$ laser performance to the use of ospemifene for vestibular symptoms related to GSM. Pain is one of the leading and often neglected symptoms of the menopausal genital, vulvar, and sexual aging.

Very few studies on postmenopausal dyspareunia have been focused on location and quality of pain. In hypoestrogenic patients with dyspareunia and vulvar burning/pain, the most exquisitely tender area is frequently the vulvar vestibule [14]. The vulvar vestibule, a thin band of tissue demarcating the entrance to the vagina covered by endodermal-originated mucosa, exhibits a high concentration of sensory free ends with a dense and shallow ramification. Vestibular pain is principally related to differences in nerve density between vagina and vulvar vestibule, and many post-menopausal women with complaints of dyspareunia have vestibular tenderness with more pronounced atrophic changes in this region rather than in the vagina [15].

In our study, it is important to highlight that vestibular dryness was significantly lower in the ospemifene+laser group compared with the same symptom in the laser group and it was already manifest after the first laser session (four weeks). The dyspareunia reduction was also higher in the ospemifene+laser group and although the improvement exhibits a lesser extent than vestibular dryness, its onset was more rapid to establish.

Ospemifene has multiple tissue-specific actions, with primarily the ability to generate a mucification and a beneficial shift of the maturation index in the vaginal mucosa [16]. The beneficial effects of ospemifene in increasing the water content of the skin are also attributed to enhanced mucopolysaccharide and hyaluronic acid production in the dermis, as a results of estrogen receptors stimulation [17]. The synthesis of the hyaluronic acid in the dermis increases, and hence the water content and hydroscopic properties of collagen increase by ospemifene therapy. The 
rationale for the pretreatment with ospemifene comes from the fact that the laser has high absorption in water and a highly moist environment allows the laser to be more effective. Another strength of our study is related to improvement of vestibular trophism in both groups even if it was faster and progressive in the ospemifene+laser group. We can speculate that ospemifene has a steering effect on regeneration and remodeling of collagen, elements that are consistent with previous studies, which showed improvement in vestibular physical examination findings in menopausal women with dyspareunia using ospemifene, as documented on vulvoscopic photography. These changes were consistent with improvements in subject-reported pain and sexual function $[18,19]$. It is also important to acknowledge that regenerative fractional laser therapy has most probably a different mechanism of action than ospemifene, resulting in induced vessel formation, reconstitution of the lamina propria and consequent regeneration of the mucosa, issue in which the ospemifene action prevails [20]. As mentioned above, dyspareunia and vaginal dryness are present simultaneously in approximately $80 \%$ of women and the parallel response of these two symptoms to ospemifene+laser clearly indicates that the anatomical and functional cause of the vestibular disorder is corrected by a synergistic action on trophism and nerve fibers hypersensitivity. As in previous studies of ospemifene, hot flush was the most commonly reported adverse effects [21]. However, considering the age and proximity to menopause of many women in the study, hot flush rates were low, and no women discontinued treatment due to this adverse effect. This trial has some limitations that are worth mentioning. Although this was a retrospective study that may introduce selection bias, all patients included have strict criteria related to GSM. Other limitations of this trial include the lacks long-term follow up and a no validated score to assess vestibular atrophy, even though used effectively in many studies. Despite the limitations, this was the first study comparing the fractional $\mathrm{CO} 2$ laser performance with the use of ospemifene for vestibular symptoms related to GSM. Based on our results we think that a combination regimen seems a valid strategy to resolve the problems related to GSM. More research is needed to understand the mechanism of action of different therapies in improving the quality of results in this underrecognized and undertreated condition.

\section{Disclosure statement}

The authors reported no conflicts of interest relevant to the study.

\section{Funding}

Medical writing editorial support was provided by Trial Form Support, with financial support provided by Shionogi Ltd. 


\section{References}

[1] Nappi RE, Kokot-Kierepa M. Vaginal Health: Insights, Views \& Attitudes (VIVA) - results from an international survey. Climacteric. 2012;15:36-44.

[2] Parish SJ, Nappi RE, Krychman ML, et al. Impact of vulvovaginal health on postmenopausal women: a review of surveys on symptoms of vulvovaginal atrophy. Int J Womens Health. 2013;5:437-447.

[3] Portman DJ, Gass MLS. Vulvovaginal Atrophy Terminology Consensus Conference Panel. Genitourinary syndrome of menopause: new terminology for vulvovaginal atrophy from the International Society for the Study of Women's Sexual Health and the North American Menopause Society. Menopause. 2014;21(10):1063-1068.

[4] Castelo-Branco C, Biglia N, Nappi RE, et al. Characteristics of postmenopausal women with genitourinary syndrome of menopause: Implications for vulvovaginal atrophy diagnosis and treatment selection. Maturitas. 2015;81(4):462-469.

[5] Faubion SS, Sood R, Kapoor E. Genitourinary syndrome of menopause: management strategies for the clinician. Mayo Clin Proc. 2017; 92(12):1842-1849.

[6] The 2017 hormone therapy position statement of The North American Menopause Society. Menopause. 2018;25:1362-1387.

[7] ACOG. Practice Bulletin No. 141: management of menopausal symptoms. Obstet Gynecol. 2014;123:202-216.

[8] Bachman G, Santen R. Treatment of vaginal atrophy. Waltham (MA): Up To Date; 2014.

[9] Portman D, Palacios S, Nappi RE, et al. Ospemifene, a non-oestrogen selective oestrogen receptor modulator for the treatment of vaginal dryness associated with postmenopausal vulvar and vaginal atrophy: a randomised, placebo-controlled, phase III trial. Maturitas. 2014;78(2): 91-98.

[10] Simon J, Portman D, Mabey RG, et al. Long-term safety of ospemifene (52-week extension) in the treatment of vulvar and vaginal atrophy in hysterectomized postmenopausal women. Maturitas. 2014; 77(3):274-281.

[11] Athanasiou S, Pitsouni E, Falagas ME, et al. CO2-laser for the genitourinary syndrome of menopause. How many laser sessions? Maturitas. 2017;104:24-28.

[12] Salvatore S, Athanasiou S, Candiani M. The use of pulsed CO2 lasers for the treatment of vulvovaginal atrophy. Curr Opin Obstet Gynecol. 2015;27(6):504-508. 
[13] Murina F, Karram M, Salvatore S, et al. Fractional CO2 laser treatment of the vestibule for patients with vestibulodynia and genitourinary syndrome of menopause: a pilot study. J Sex Med. 2016;13(12): 1915-1917.

[14] Lev-Sagie A. Vulvar and vaginal atrophy: physiology, clinical presentation, and treatment considerations. Clin Obstet Gynecol. 2015; 58(3):476-491.

[15] Graziottin A, Murina F. Vulvar Pain: From Childhood to Old Age [Internet]. Springer International Publishing; 2017. [cited 2019 May 22]. Available from: https://www.springer.com/gp/book/9783319426754.

[16] Taras TL, Wurz GT, DeGregorio MW. In vitro and in vivo biologic effects of Ospemifene (FC-1271a) in breast cancer. J Steroid Biochem Mol Biol. 2001;77(4-5):271-279.

[17] Brincat MP, Baron YM, Galea R. Estrogens and the skin. Climacteric. 2005;8(2):110-123.

[18] Goldstein SW, Winter AG, Goldstein I. Improvements to the vulva, vestibule, urethral meatus, and vagina in women treated with ospemifene for moderate to severe dyspareunia: a prospective vulvoscopic pilot study. Sex Med. 2018;6(2):154-161.

[19] Murina F, Di Francesco S, Oneda S. Vulvar vestibular effects of ospemifene: a pilot study. Gynecol Endocrinol. 2018;34(7):631-635.

[20] Fitzpatrick RE, Rostan EF, Marchell N. Collagen tightening induced by carbon dioxide laser versus erbium: YAG laser. Laser Surg Med. 2000;27(5):395-403.

[21] Nappi RE, Panay N, Bruyniks N, et al. The clinical relevance of the effect of ospemifene on symptoms of vulvar and vaginal atrophy. Climacteric. 2015;18(2):233-240. 
Table 1. Demographic and clinical characteristics of study participants at baseline.

\begin{tabular}{|c|c|c|c|}
\hline & $\begin{array}{l}+ \text { Ospemifene group } \\
\qquad(\mathrm{n}=39)\end{array}$ & $\begin{array}{l}\text { Laser group } \\
(\mathrm{n}=33)\end{array}$ & $\mathrm{P}$ \\
\hline Age (years) & $56.0 \pm 6.1$ & $55.1 \pm 5.9$ & NS \\
\hline Years since menopause & $6.1 \pm 3.4$ & $6.3 \pm 3.3$ & NS \\
\hline BMI & $22.9 \pm 3.9$ & $23.7 \pm 3.3$ & NS \\
\hline Duration of symptoms (months) & $4.3 \pm 2.9$ & $4.6 \pm 2.8$ & NS \\
\hline \multicolumn{4}{|l|}{ VAS } \\
\hline Dryness & $6.5 \pm 2.4$ & $7.3 \pm 2.1$ & NS \\
\hline Burning/Pain & $4.9 \pm 3.7$ & $4.1 \pm 2.9$ & NS \\
\hline Dyspareunia & $7.3 \pm 2.7$ & $8.6 \pm 2.5$ & NS \\
\hline Vestibular health score ${ }^{a}$ & $11.5 \pm 6.4$ & $12.3 \pm 5.8$ & NS \\
\hline \multicolumn{4}{|c|}{ Data are presented as the mean values \pm standard deviation. } \\
\hline \multicolumn{4}{|c|}{$\begin{array}{l}\text { BMI: Body Mass Index; VAS: Visual Analog Scale }(0-10 \text {, where } 0=\text { no symptom and } 10=\text { severe } \\
\text { symptom). }\end{array}$} \\
\hline \multicolumn{4}{|c|}{${ }^{a}$ Observations for petechiae, pallor, friability, dryness, and redness in the mucosa. Ratings were } \\
\hline
\end{tabular}


Table 2. Severity of GSM Symptoms at baseline, weeks 4, 8, 12 after treatment and at final followup at 3 months.

$$
\text { Baseline } \quad 4 \text { weeks } \quad 8 \text { weeks } \quad 12 \text { weeks } \quad 3 \text { months }
$$

Ospemifene+Laser group $(\mathrm{n}=39)$

\begin{tabular}{|c|c|c|c|c|c|}
\hline Dryness & $6.5 \pm 2.4$ & $\begin{array}{l}2.3 \pm 2.3 \\
(\mathrm{p}<.01)\end{array}$ & $\begin{array}{l}2.6 \pm 1.8 \\
(\mathrm{p}<.01)\end{array}$ & $\begin{array}{l}1.3 \pm 1.1 \\
(\mathrm{p}<.01)\end{array}$ & $\begin{array}{l}0.8 \pm 1.2 \\
(\mathrm{p}<.01)\end{array}$ \\
\hline Burning/Pain & $4.9 \pm 3.7$ & $\begin{array}{l}3.2 \pm 3.4 \\
(\mathrm{NS})\end{array}$ & $\begin{array}{l}2.9 \pm 2.1 \\
(\mathrm{p}<.001)\end{array}$ & $\begin{array}{l}1.9 \pm 1.1 \\
(\mathrm{p}<.001)\end{array}$ & $\begin{array}{l}1.7 \pm 2.1 \\
(p<.001)\end{array}$ \\
\hline Dyspareunia & $7.3 \pm 2.7$ & $\begin{array}{l}6.3 \pm 2.1 \\
(\mathrm{NS})\end{array}$ & $\begin{array}{l}5.6 \pm 2.9 \\
(\mathrm{p}<.01)\end{array}$ & $\begin{array}{l}2.3 \pm 1.7 \\
(\mathrm{p}<.01)\end{array}$ & $\begin{array}{l}1.3 \pm 2.2 \\
(p<.01)\end{array}$ \\
\hline
\end{tabular}

Laser group $(\mathrm{n}=33)$

$\begin{array}{llllll}\text { Dryness } & 7.3 \pm 2.1 & 5.3 \pm 2.8 & 5.6 \pm 1.7 & 4.3 \pm 2.1 & 4.8 \pm 2.2 \\ & & (\mathrm{p}<.01) & (\mathrm{p}<.01) & (\mathrm{p}<.01) & (\mathrm{p}<.01) \\ \text { Burning/Pain } & 4.1 \pm 2.9 & 3.9 \pm 2.5 & 2.8 \pm 1.9 & 1.8 \pm 1.1 & 1.9 \pm 1.9 \\ & & (\mathrm{NS}) & (\mathrm{p}<.01) & (\mathrm{p}<.01) & (\mathrm{p}<.01) \\ \text { Dyspareunia } & 8.6 \pm 2.5 & 6.9 \pm 2.4 & 6.6 \pm 2.7 & 4.3 \pm 1.9 & 3.1 \pm 1.9 \\ & & (\mathrm{p}<.01) & (\mathrm{p}<.01) & (\mathrm{p}<.01) & (\mathrm{p}<.01)\end{array}$

The values present mean \pm standard deviation.

NS: Statistically non-significant p-value.

Statistical significance from baseline was determined using student's t-test.

$P$ values $<.05$ were considered statistically significant

Table 3. Results of the vestibular health score for both groups.

\begin{tabular}{llllll} 
& Baseline & 4 weeks & 8 weeks & 12 weeks & 3 months \\
\hline Ospemifene+Laser & $11.5 \pm 6.4$ & $8.3 \pm 3.3$ & $6.6 \pm 3.8$ & $4.4 \pm 2.7$ & $3.8 \pm 1.2$ \\
group (n=39) & & $(\mathrm{p}<.01)$ & $(\mathrm{p}<.01)$ & $(\mathrm{p}<.01)$ & $(\mathrm{p}<.01)$ \\
Laser group (n=33) & $12.3 \pm 5.8$ & $11.2 \pm 4.4$ & $9.9 \pm 4.1$ & $4.3 \pm 2.1$ & $4.7 \pm 2.2$ \\
& & $(\mathrm{NS})$ & $(\mathrm{p}<.01)$ & $(\mathrm{p}<.01)$ & $(\mathrm{p}<.01)$
\end{tabular}

Observations for petechiae, pallor, friability, dryness, and redness in the mucosa.

Ratings were based on a 4-point scale (0: none; 1: mild; 2: moderate;3: severe).

Statistical significance from baseline was determined using student's t-test.

$\mathrm{p}<.05$ is considered statistically significant. 

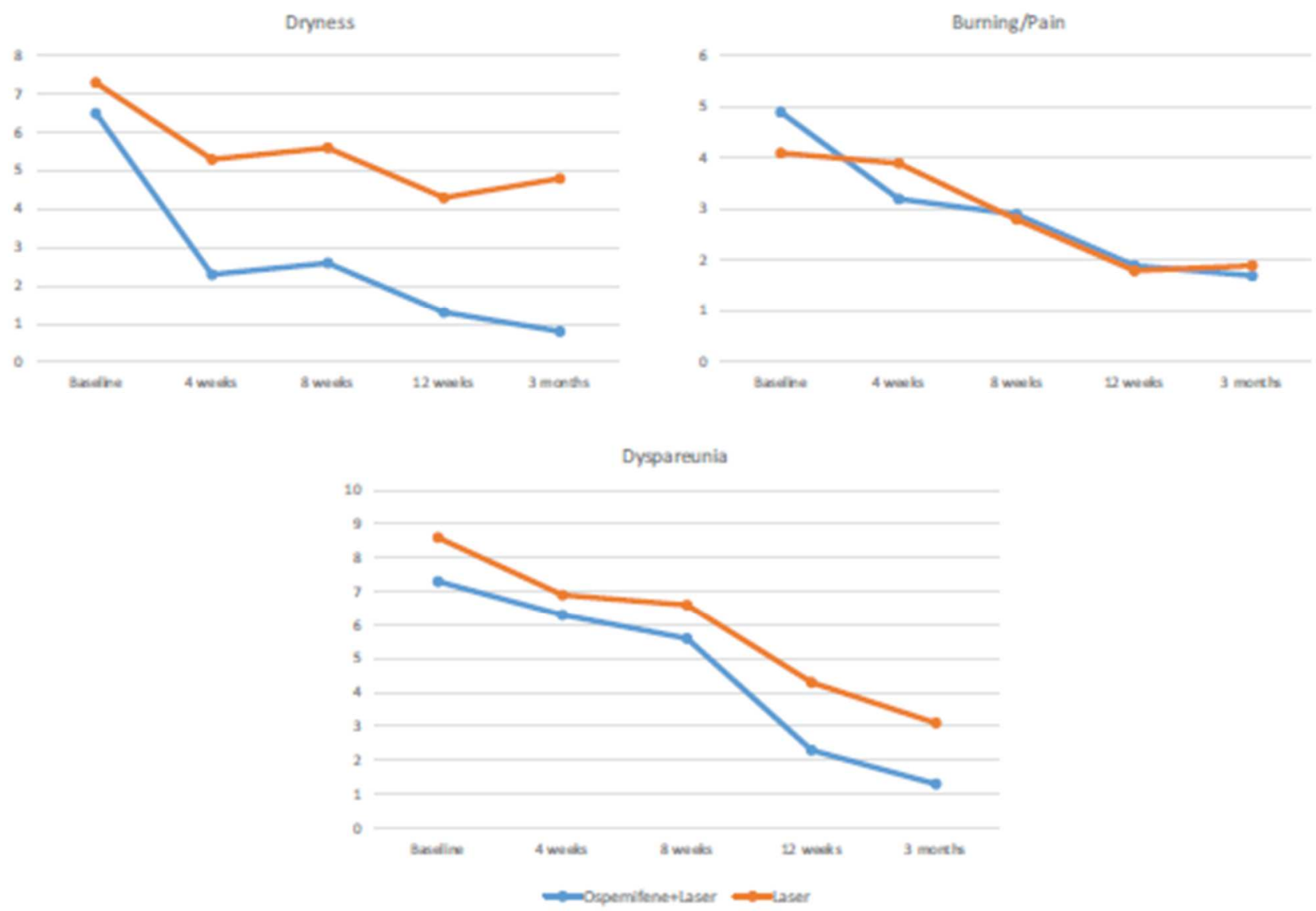

Figure 1. Assessment of the following symptoms of GSM: (a) dyspareunia, (b) dryness, (c) burning/pain on a 0-10 VAS scale in the ospemifene+laser group and in the laser group before the treatment and after 4, 8, 12 weeks and 3 months.

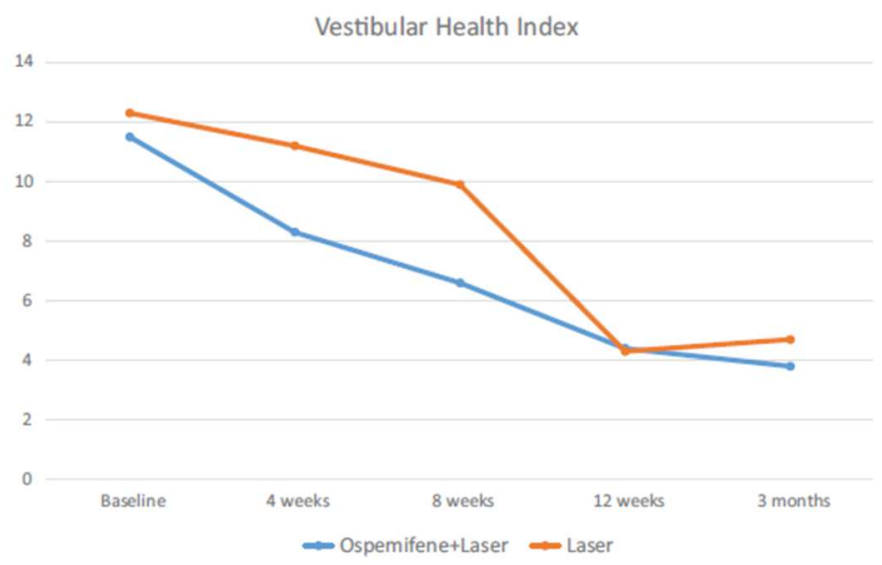

Figure 2. Assessment of vestibular health score for both groups (observations for petechiae, pallor, friability, dryness, and redness in the mucosa. Ratings were based on a 4-point scale -0: none; 1 : mild; 2: moderate; 3: severe). 